\title{
Tangence
}

\section{La revendication du réel : le cas de la poésie intime}

\section{Hélène Marcotte}

Numéro 45, octobre 1994

Authenticité et littérature personnelle

URI : https://id.erudit.org/iderudit/025824ar

DOI : https://doi.org/10.7202/025824ar

Aller au sommaire du numéro

Éditeur(s)

Tangence

ISSN

0226-9554 (imprimé)

1710-0305 (numérique)

Découvrir la revue

Citer cet article

Marcotte, H. (1994). La revendication du réel : le cas de la poésie intime.

Tangence, (45), 50-60. https://doi.org/10.7202/025824ar d'utilisation que vous pouvez consulter en ligne.

https://apropos.erudit.org/fr/usagers/politique-dutilisation/ 


\section{La revendication du réel: le cas de la poésie intime}

\section{Hélène Marcotte}

"[U]n écrivain en dit plus, et plus vrai, quand il "ment", c'està-dire quand il fictionne, que lorsqu'il vise un dévoilement " ${ }^{1}$. Cette affirmation de Jean Bellemin-Noël non seulement invite les lecteurs à établir une distinction radicale entre la littérature dite intime et les œuvres de fiction mais elle les incite à rechercher l'intime là où l'auteur choisit de l'évincer ou, à tout le moins, de le travestir. Le cas de la poésie intime est toutefois particulier. Considérée par les uns comme une pure fiction, par les autres comme un document du réel, la poésie intime pourrait se situer, suivant une troisième perspective, à la frontière de ces deux catégories de textes. Qu'en est-il réellement?

Lire délibérément l'œuvre poétique d'un auteur dans une perspective autobiographique est chose courante. En effet, si les auteurs ne cèdent pas toujours à la tentation autobiographique, peu de lecteurs le conçoivent ainsi et il n'est pas rare que la biographie d'un poète soit entrecoupée de citations puisées à même son œuvre. Est-ce à dire que poème intime est synonyme de poème autobiographique ou, mieux encore, d'autobiographie versifiée? Lorsque le poème se déploie à la première personne, l'équation auteur $=$ narrateur $=$ personnage soulève peu d'objections. La coïncidence entre l'auteur (la personne réelle qui écrit le texte), le narrateur (la personne qui parle à l'intérieur du texte ou le sujet de l'énonciation) et le personnage (la personne dont on parle ou le sujet de l'énoncé) semble aller de soi. Pourtant, "l'expression je crée aussi peu la position je que le démonstratif ceci ne crée le spectacle du monde vers lequel pointe l'indicateur déictique "2. Affirmer, comme le fait Émile Benveniste, que "je est l'individu qui énonce la présente instance du discours contenant

1 Jean Bellemin-Noël, "Les droits du lecteur sur son texte", Paragrapbes, Montréal, Département d'études françaises, $\mathrm{n}^{\circ} 3$ (1989-1990), p. 102.

2 Paul Ricœur, "La question du sujet: le défi de la sémiologie", Le conflit des interprétations, Paris, Seuil, 1969, p. 252. 
je" 3 appelle d'autres questions. Qu'est-ce qui distingue le "je" d'un roman de celui d'une autobiographie? Où situer celui d'un poème écrit à la première personne? C'est à cette dernière question que nous aimerions répondre, en puisant nos exemples dans la poésie québécoise des $\mathrm{XvII}^{\mathrm{e}}$ et $\mathrm{XIX}^{\mathrm{e}}$ siècles.

\section{Entre fiction et réalité}

Dans ses Principes de littérature, ouvrages reprenant les canons d'une poétique traditionnelle et servant de livres de référence au Séminaire de Québec, entre autres, l'abbé Charles Batteux prend le parti de la fiction et tente d'établir une équivalence entre les trois genres poétiques reconnus, l'épique, le dramatique et le lyrique ${ }^{4}$, en soumettant le lyrisme au principe d'imitation:

De même donc que dans la poésie épique et dramatique on imite les actions et les mœurs, de même dans le lyrique, on chante les sentimens, ou les passions imitées. S’il y a du réel, il se mêle avec ce qui est feint, pour faire un tout de même nature: la fiction embellit la vérité, et la vérité donne du crédit à la fiction. ${ }^{5}$

Dans une note ajoutée en 1764 à ses Principes, l'abbé Batteux revient sur le rôle de l'imitation dans la poésie lyrique et essaie de contrer les objections de son traducteur, Johann Adolf Schlegel, qui soutient que, très souvent, le poète chante des sentiments réels et non des sentiments imités:

Je n'avais à y prouver que deux choses: la première, que les sentimens peuvent être feints et imités comme les actions; [...] la seconde, que tous les sentimens exprimés dans le lyrique, feints ou vrais, devaient être soumis aux règles de l'imitation poëtique, c'est-à-dire, qu'ils doivent être vraisemblables, choisis, soutenus, aussi parfaits qu'ils peuvent l'être en leur genre, et enfin rendus avec toutes les grâces et toute la force de l'expression poétique. C'est le sens du principe de l'imitation, c'en est l'esprit. ${ }^{6}$

3 Émile Benveniste, Problèmes de linguistique générale, Paris, Gallimard, 1965, p. 259.

4 Notons que quelques auteurs reconnaissent un quatrième genre, le genre didactique.

5 L'abbé [Charles] Batteux, Principes de litttérature, t. I, Paris, Bellavoine, 1824, p. 268-269.

6 Ibid., p. 260. 
52

L'intégration de la poésie lyrique au principe d'imitation ne s'effectue pas, comme on peut le voir, sans qu'un détournement de sens ne s'opère et ne conduise à une généralisation abusive : il ne s'agit plus d'une "simple possibilité d'expression fictive mais d'une fictivité essentielle des sentiments exprimés" 7 . Ainsi, en faisant de l'imitation une des caractéristiques intrinsèques de la poésie lyrique, Batteux la rattache à la fiction. Mais alors que pour Batteux il suffit que les sentiments exprimés par l'auteur puissent être feints pour que le genre lyrique tout entier soit soumis au principe d'imitation, pour Schlegel, la possibilité que les sentiments soient réels lui permet d'échapper à ce principe. Le problème reste donc entier, la classification de la poésie lyrique dépendant du parti pris des auteurs des traités.

Käte Hamburger, dans la Logique des genres littéraires, remplace la triade chère aux classiques par le genre fictionnel ou mimétique (littérature épique ou narrative et littérature dramatique), le genre lyrique (poésie lyrique) et le genre mixte (ballade, roman à la première personne). Cette nouvelle répartition des genres littéraires s'articule à partir d'une dichotomie fondamentale: les formes qui relèvent de la fiction se caractérisent par une énonciation fictive qui se rapporte aux personnages, les Je-Origines fictifs, tandis que celles qui relèvent du lyrique se fondent sur une énonciation "historique" ou référentielle, c'est-à-dire qui se rapporte à l'auteur, le Je-Origine réel, sujet authentique que l'on peut identifier et situer dans le temps. Il va sans dire que les genres mixtes entremêlent ces deux modes d'énonciation.

Selon Hamburger, alors que le lecteur perçoit le caractère illusoire des énoncés dès les premières pages d'un roman, il reçoit le poème lyrique comme un "énoncé de réalité " puisque c'est le poète qui prend la parole, et non un personnage fictif. Cependant, la coïncidence de l'auteur du poème lyrique avec un Je-Origine réel n'affecte que l'énonciation. Le lecteur conserve la possibilité d'accepter ou de nier la véracité de l'énoncé, rien n'indiquant que le contenu du poème doive s'inspirer de faits vécus:

Le sujet d'énonciation lyrique s'identifie donc avec le poète, exactement comme celui d'une ouvre historique, philosophique ou scientifique s'identifie avec son auteur, au sens logi-

7 Gérard Genette, Introduction à l'arcbitexte, Paris, Seuil, 1979, p. 38. 
que du terme. Mais alors que, dans le cas de ces documents de réalité, cette identité n'est pas problématique puisque, seul l'objet étant visé, le sujet d'énonciation n'a pas de rôle par rapport au contenu, il en va autrement avec le Je lyrique. L'identité logique ne signifie pas, dans ce cas, que tous les énoncés d'un poème, ou même le poème dans son entier, doivent correspondre à une expérience réelle de l'auteur [...]. ${ }^{8}$

Pour Hamburger, menteur, rêveur ou fabuliste, l'auteur de poésies lyriques n'en demeure pas moins sujet d'énonciation: "Certes, l'expérience peut être "fictive" au sens d'invention, mais le sujet de l'expérience, et, avec lui, le sujet d'énonciation, le Je lyrique, ne peut être que réeln'. Puisque l'identification entre le poète et le Je lyrique ne s'effectue que d'un point de vue logique, il apparaît impossible à Hamburger d'établir de lien biographique entre les deux; l'identification - éventuelle ou effective - de l'auteur et du narrateur-personnage reste dans l'indistinction:

Il n'y a pas de critère, ni logique ni esthétique, ni interne ni externe, qui nous autoriserait à dire si le sujet d'énonciation du poème peut être ou non identifié avec le poète. Nous n'avons la possibilité, et donc le droit, ni de soutenir que le poète présente ce qu'énonce le poème - que ce soit ou non sous la forme d'un Je - comme étant sa propre expérience, ni d'affirmer le contraire. ${ }^{10}$

Pour intéressante qu'elle soit, cette nouvelle façon d'envisager la question des genres, et plus particulièrement la question du lyrisme, puisqu'elle laisse dans l'ombre les rapports entre l'énoncé et le sujet de l'énonciation, ne permet pas d'établir des critères probants pour la constitution d'un corpus. Cette fois, c'est le parti pris des lecteurs qui détermine la part de la fiction et du réel dans les textes.

Dans Le pacte autobiograpbique, Philippe Lejeune précise le lien qui unit les trois instances narratives et maintient que "[p]our

8 Käte Hamburger, Logique des genres littéraires, Paris, Seuil, 1986, p. 241.

9 Ibid., p. 243.

10 Ibid., p. 240. Hamburger ajoute à la page 245: "Ce que Gothe, pour décrire sa propre expérience, a formulé en disant: "aucun trait ne figure qui n'ait été vécu, mais aucun trait ne figure tel qu'il a été vécu "vaut, avec des nuances, pour toute la poésie lyrique, c'est-à-dire pour toute expérience vécue transformée en poème. Ce mot de Gœthe, en outre, interdit deux choses: nier l'identité entre le Je lyrique et le Je du poète, décréter l'identité entre ce qui est énoncé et mis en forme par le poète et l'expérience "réelle" ". 
qu'il y ait autobiographie (et plus généralement littérature intime), il faut qu'il y ait identité de l'auteur, du narrateur et du personnage ${ }^{11}$. Il n'hésite pas d'ailleurs à reconnaître cette identité dans ce qu'il appelle le "poème autobiographique", lequel pourrait se définir comme un récit rétrospectif en vers qu'une personne réelle fait de sa propre existence, lorsqu'elle met l'accent sur sa vie individuelle, en particulier sur l'histoire de sa personnalité ${ }^{12}$. Lejeune en vient toutefois à nuancer sa position lorsqu'il affirme qu'il faut situer de tels textes "dans leur différence, aussi bien par rapport à la poésie (emploi d'un "je " nettement autobiographique gagé sur le nom propre de l'auteur, à la place du "je "lyrique traditionnel) que par rapport à l'autobiographie" 13 . Mais comment cette distinction se manifeste-t-elle au lecteur de poèmes? $\mathrm{Si}$ "[l]e genre se définit encore par l'“intentionnalité", l'“attitude naturelle" de la conscience de l'écrivain, comme du lecteur [...]"14, comment le lecteur, indépendamment de son intention de lecture, peut-il percevoir celle de l'auteur du poème lyrique et saisir la nature du contrat de lecture proposé?

Avant même d'amorcer la lecture d'un ouvrage, les marques paratextuelles permettent au lecteur d'identifier le type de texte qu'il a sous les yeux, l'indication générique "poésies" apparaissant habituellement sur la couverture ou sur la page de titre. Cette indication seule ne suffit pas cependant à établir un contrat de lecture et à rejeter les textes du côté de la fiction ou du côté de l'autobiographie, le poème se définissant laconiquement comme un "ouvrage en vers". Dès lors, la signature qui apparaît au bas du texte poétique constitue-t-elle un "pacte autobiographi-

11 Philippe Lejeune, Le pacte autobiograpbique, Paris, Seuil, 1975, p. 15.

12 Cette définition reprend en fait la définition de l'autobiographie que donne Lejeune, mais dans laquelle "en vers" est mis pour "en prose", et s'appuie sur ce que Lejeune lui-même en dit (voir Le pacte autobiographique, p. 14). Pour notre part, nous serions d'avis que ce genre de poème, dont The Prelude de Wordsworth ou encore Chêne et Cbien de Raymond Queneau pourraient servir d'exemples, devrait plutôt prendre l'appellation d'uautobiographie versifiée". Nous réservons le terme de poème autobiographique pour des poèmes tels "L'Expatrié. Dans l'infortune" de N. D. J. Jeaumenne (voir supra), dans lesquels lauteur ne coincide pas avec le narrateur-personnage bien qu'il partage certains traits avec lui.

13 Philippe Lejeune, "Le Pacte autobiographique (bis)", dans L'autobiographie en Espagne, Publications de l'Université de Provence, 1982, p. 18. Sur cette question, voir aussi Philippe Lejeune, Le pacte autobiographique, p. 245-246.

14 Dominique Combe, Les genres littéraires, Paris, Hachette, 1992, p. 15. 
que" au sens où l'entend Lejeune ${ }^{15}$ ? En signant son poème, l'auteur revendique-t-il la responsabilité de l'énonciation du texte et, du fait même, le contenu de l'énoncé ou se contente-t-il de s'attribuer la composition dudit texte? Dans la plupart des cas, s'il est certain que l'auteur assume le poème en tant que bien symbolique ${ }^{16}$, rien n'indique qu'il en assume nécessairement le discours, du moins, il ne l'assume pas explicitement ou, si l'on préfère, textuellement. C'est d'ailleurs pourquoi l'usage très répandu de l'anonymat et du pseudonymat tout au long du $\mathrm{XIX}^{\mathrm{e}}$ siècle ne pose pas véritablement problème: la véracité des dires de l'auteur ne peut être vérifiée, et n'a pas à l'être, comme c'est le cas dans les genres intimes consacrés (biographie, autobiographie, mémoires, etc.). Il nous importe peu de savoir si effectivement l'auteur du poème a vécu la situation qu'il décrit, s'il a réellement ressenti les sentiments exprimés ou s'il s'agit en fait d'un exercice de versification mettant en scène un "je" fictif. Selon nous,

le propre de la poésie "lyrique" réside bien plutôt dans une énonciation foncièrement ambivalente. La référence du JE lyrique est un mixte indécidable d'autobiographie et de fiction. En d'autres termes, dans le poème lyrique, le pronom JE, certes dominant, réfère simultanément et indissociablement à une figure "réelle", historique, biographique, du poète en tant que personne, et à une figure entièrement construite, fictive. ${ }^{17}$

Ici, seul le critère de la vraisemblance peut s'appliquer. Aussi, lorsque rien ne vient infirmer que le "je "qui parle et dont on parle renvoie à l'auteur, nous considérerons que le critère de vraisemblance est respecté et que le poème, s'il remplit les autres conditions, peut être qualifié d'“intime " 18 .

\section{Le critère de vraisemblance}

Il est fréquent dans les poèmes intimes que non seulement le critère de vraisemblance soit respecté, mais que l'auteur et le

15 Voir Le pacte autobiographique, p. 26: "Le pacte autobiographique, c'est l'affirmation dans le texte de cette identité, l'identité du nom (auteur-narrateur-personnage)] renvoyant en dernier ressort au nom de l'auteur sur la couverture".

16 Voir Pierre Bourdieu, "Le marché des biens symboliques", L'année sociologique, vol. III, $\mathrm{n}^{\circ} 22$ (1971), p. [49]-126.

17 Dominique Combe, Poésie et récit, une rbétorique des genres, op. cit., p. 162.

18 Comme nous le verrons plus loin, dans notre propos, les termes "poème lyrique "et "poème intime" ne sont pas synonymes. 
narrateur-personnage partagent certains ou plusieurs traits biographiques. En effet, très souvent le titre du poème, la dédicace, une note en bas de page ou encore un passage du texte permettent le rapprochement entre le "je" du narrateur-personnage et le signataire du poème. Citons " $\mathrm{A}$ ma fille Jeanne à sa première communion " 19 de Louis Fréchette, "Le cimetière. À Jessy" 20 de Charles Lévesque ou encore "À mes petites soeurs Léontine et Angéline" 21 de Pamphile Lemay. Mais, de même que certains indices permettent le rapprochement entre le contenu du texte et la vie privée de l'auteur, d'autres établissent entre eux une distance telle que le texte contrevient alors au critère de vraisemblance.

L'absence d'un rapport d'identité entre le "je" de l'auteur et celui du narrateur-personnage s'établit dès que l'énonciation est attribuée à un personnage, mais la reconnaissance du personnage peut se manifester de diverses manières. Par exemple, dans le "Discours de S[imon] L[atresse] au lit de la mort"22, l'indice établissant la distance entre l'auteur et le narrateur-personnage n'est pas inscrit dans le texte. D'entrée de jeu, le titre du poème porte à croire que Simon Latresse assume l'énonciation du poème. L'absence de signature et l'attribution du poème à Joseph Quesnel introduisent cependant un doute dans l'esprit du lecteur. Un entrefilet du Canadien relate les circonstances de la mort du jeune homme: le 12 septembre 1807, des soldats britanniques tentent d'enrôler de force quelques Canadiens dans la marine; alors qu'il essaie de s'échapper, le jeune Latresse est tiré à bout portant et meurt le lendemain. Le poète s'inspire donc d'un fait réel et met dans la bouche de Simon Latresse les sentiments qu'il aurait pu ressentir au moment de l'agonie. Sitôt l'anecdote con-

19 Louis Fréchette, "À ma fille Jeanne à sa première communion", Épaves poétiques, Montréal, Librairie Beauchemin, 1908, p. 107.

20 Charles Lévesque, "Le Cimetière. À Jessy", L'écbo du Cabinet de lecture paroissial (12 décembre 1846), p. 1, reproduit dans Les ceutres de Charles Lévesque écrivain oublié du dix-neuvième siècle (1817-1859), texte établi et annoté par Michel Boucher, thèse de maitrise, Québec, Université Laval, 1972 , p. 72 , et dans Les textes poétiques du Canada français, tome IV, Montréal, Fides, 1991, n ${ }^{\circ} 367$, p. 745-746.

21 Léon Pamphile Lemay, "À mes petites sœurs Léontine et Angéline", Essais poétiques, Québec, G. E. Desbarats, 1865, p. 179.

22 (Joseph Quesnell, "Discours de S[imon] L[atressel au lit de la mort ", Le canadien (3 octobre 1807), p. 182, reproduit dans TPCF, tome II, Montréal, Fides, 1989, no 35, p. 60. Sur le drame, voir Le canadien (19 septembre 1807), p. 182 . 
nue du lecteur, la part de la fiction ne fait plus aucun doute. Bien qu'il se déroule à la première personne et que le narrateur, en plus de coïncider avec le personnage principal, exprime des sentiments personnels, le texte ne peut être qualifié d'intime car le critère de vraisemblance n'est pas respecté. Nous savons de façon certaine que l'auteur ne coïncide pas avec le narrateurpersonnage.

Dans la "Chanson" 23 de Marie-Marguerite La Corne, c'est le sexe masculin du narrateur-personnage qui empêche l'identification à l'auteure. L'inverse se produit dans les poèmes de JosephGuillaume Barthe, signés du pseudonyme "Marie-Louise" et publiés entre le 10 mai et le 6 novembre 1837 . Ces poèmes sont à l'origine d'un échange amoureux entre Joseph-Guillaume Barthe (Marie-Louise), N. D. J. Jeaumenne (Le solitaire) et AndréRomuald Cherrier (Pierre-André). Sitôt la véritable identité de Marie-Louise dévoilée, les poèmes de Barthe perdent leur statut d'intimes puisque l'auteur, de sexe masculin, ne peut plus être identifié à la narratrice-personnage. Toutefois, les poèmes de Jeaumenne et de Cherrier le demeurent: seul le destinataire de leurs poèmes a changé; l'auteur-narrateur-personnage renvoie toujours à une même personne et les sentiments exprimés n'en sont pas moins intimes et profonds du fait qu'ils s'adressent à une personne imaginaire.

Très souvent, au XIX ${ }^{e}$ siècle, c'est le titre du poème qui marque la distance entre l'auteur et le narrateur-personnage en indiquant la source de l'énonciation. Ainsi, dans des poèmes comme "Le berger malheureux" 24 attribué à Augustin-Norbert Morin, "Le voltigeur de retour " ${ }^{25}$ de François-Xavier Garneau, "L'Africaine " 26

23 Marie-Marguerite La Corne, "Chanson", Ma saberdache, vol. 1, Correspondance, lettre de $\mathrm{M}^{\text {me }}$ Viger à Jacques Viger, Québec, datée de Montréal, le 13 février 1809, p. 143-144, reproduit dans TPCF, tome II, op. cit., no 50 , p. 89-90.

24 [Augustin-Norbert Morin], "Le berger malheureux", Le canadien (20 décembre 1820), p. 390, reproduit dans TPCF, tome II, op. cit., no 282, p. 524-525.

25 François-Xavier Garneau, "Le voltigeur de retour", Le canadien (2 juillet 1831), p. 1, reproduit dans TPCF, tome III, Montréal, Fides, 1990, $\mathrm{n}^{\circ} 112$, p. 168-169.

26 Charles Lévesque, "L'Africaine", Le moniteur canadien (1 ${ }^{\mathrm{er}}$ avril 1852), p. 1, reproduit dans Les auvres de Charles Lévesque écrivain oublié du dix neuvième siècle (1817-1859), texte établi et annoté par Michel Boucher, op. cit., p. 124, et dans TPCF, tome V, Montréal, Fides, 1992, n 70, p. 149-151. 
de Charles Lévesque, ou encore "La nymphe de la fontaine " ${ }^{27}$ de Louis Fréchette, la prise en charge du discours se fait par le personnage identifié dans le titre. Le "je" qui parle renvoie à ce personnage, donc à un "je " fictif. Aussi, la présence de personnages qui prennent en charge l'énonciation à l'intérieur du poème infléchit le genre vers la fiction tandis que l'absence de tels procédés le rapproche de l'autobiographie.

On pourrait objecter ici la possibilité que l'auteur ait choisi d'utiliser un personnage intermédiaire pour traduire ses sentiments intimes. La question qui se pose alors est de savoir si l'on reste toujours dans le registre personnel. L'utilisation d'un personnage, qu'il y ait changement ou non de personne grammaticale, entraîne dans certains cas une ambiguilté. Qui parle? Dans des poèmes-dialogues, comme Soir d'automne ${ }^{28}$ de James-E.-P. Prendergast ou encore La consolatrice ${ }^{29}$ de J. B. de Flandre, pseudonyme-anagramme d'Albert Ferland, les auteurs mettent en scène un poète et sa muse. L'utilisation du "je", la situation analogique entre le narrateur-personnage et l'auteur accentuent alors la confusion.

L'utilisation de la troisième personne soulève à l'occasion des problèmes similaires ${ }^{30}$. Prenons le cas de "L'expatrié. Dans l'infortune" attribué à N. D. J. Jeaumenne. Le poème se déroule à la troisième personne de sorte qu'il ne semble pas y avoir de coïncidence entre l'auteur, le narrateur et le personnage principal:

Séparé par les mers d'une terre chérie,

C'est alors seulement qu'on aime sa patrie!

C'est alors que, rempli d'aimables souvenirs,

Le cour de l'exilé se gonfle de soupirs

Que ses regrets, mêlés d'une douce tristesse

Font couler de ses yeux des larmes de tendresse.

Souvent dans un doux songe et pour lui plein d'attraits,

D'une mère chérie il croit revoir les traits [...]. ${ }^{31}$

27 Louis Fréchette, "La nymphe de la fontaine ", Mes loisirs, Montréal/Paris, Éditions Leméac/Éditions d'Aujourd'hui, 1979, p. 95.

28 James-E.-P. Prendergast, Soir d'automne, Québec, P.-G. Delisle imprimeur, $1881,21 \mathrm{p}$.

29 J. B. de Flandre, La consolatrice, Montréal, Poirier, Bessette \& Cie, 1898, 14 p.

30 Nous n'avons rencontré aucun poème canadien dans lequel le narrateurpersonnage s'interpelle à la deuxième personne.

31 [N. D. J. Jeaumenne], "L'expatrié. Dans l'infortune ", L'impartial (15 janvier 1835), p. 1, reproduit dans $T P C F$, tome III, op. cit., $\mathrm{n}^{\circ} 248$, p. 441-442. 
La biographie de Jeaumenne nous permet cependant de considérer l'Expatrié comme une figure exemplaire du poète. La fusion s'opère donc, même si l'auteur a choisi de nier ou de camoufler la ressemblance entre le sujet de l'énonciation et celui de l'énoncé. Ici, comme dans les poèmes de Prendergast et de Ferland, la tentation est grande d'inclure ces pièces dans la poésie intime. Toutefois, prendre ce parti serait présumer d'une identité entre l'auteur et le narrateur-personnage que l'auteur refuse d'assumer. Bien qu'il soit impossible de faire fi des indications biographiques qu'introduit le poète dans ses vers, la présence d'un personnage prenant en charge le discours, fût-il identifié de façon aussi vague et mimétique que "le poète", témoigne du désir de l'auteur d'universaliser sa poésie. Prendre le parti de l'intime serait fausser la réalité. Nous appellerons donc "poèmes autobiographiques" les poèmes dans lesquels il est possible d'établir une ressemblance entre l'énoncé et la biographie de l'auteur mais où il n'y a pas d'identité assumée ou "vraisemblable" entre l'auteur et le narrateur-personnage.

\section{Quelques notes sur la poésie intime}

Pour important qu'il soit, le critère de vraisemblance ne suffit pas à définir la poésie intime. En fait, pour qu'il y ait poésie intime, quatre critères doivent être respectés par le texte. Il faut:

a) Que la narration se fasse à la première personne par un même personnage tout au long du texte;

b) qu'il y ait rapport d'identité entre le narrateur et le personnage principal;

c) que rien ne vienne infirmer la possibilité que l'auteur $=$ narrateur $=$ personnage (critère de vraisemblance);

d) que la relation d'événements privés de même que l'expression des sentiments personnels et des états d'âme l'emportent sur toute autre information (dominante ${ }^{32}$ ).

Comme on peut le constater, poésie intime n'est pas synonyme dans notre propos de poésie lyrique, poésie qui, selon la plupart

32 Dominique Combe donne de la dominante la définition suivante: "en linguistique, fonction ou forme majoritaire qui gouverne un énoncé, à laquelle les autres composantes sont subordonnées". (Dominique Combe, Les genres littêraires, op. cit., p. 159). 
des définitions, "n'a pour objet que d'exprimer les sentiments" 33 . Leur seul point commun: le quatrième critère de la définition, c'est-à-dire la dominante. Ainsi, un poème qui exprime le chagrin d'une mère ayant perdu son enfant, tel "Consolation" 34 de Charles Lévesque, ou tout simplement une pièce de vers traitant de la tristesse occasionnée par la perte d'un enfant, comme le poème "Sur la mort prématurée du jeune Petro Adrien Delvecchio, décédé à six ans et deux mois" 35 de Joseph-Guillaume Barthe, peuvent être considérés comme des poèmes lyriques, parce qu'ils sont centrés sur l'expression de sentiments, mais non comme des poèmes intimes, puisqu'ils contreviennent aux autres critères et ne rendent pas compte explicitement de la subjectivité intime du poète. Or ce qui nous intéresse dans la poésie intime, c'est la façon dont l'individu définit sa relation à soi, aux autres et au monde à partir de sa propre subjectivité:

Recommencer au fond de soi le Cogito d'un écrivain ou d'un philosophe, c'est retrouver sa façon de sentir et de penser, voir comment elle nait et se forme, quels obstacles elle rencontre; c'est redécouvrir le sens d'une vie qui s'organise à partir de la conscience qu'elle prend d'elle-même. ${ }^{36}$

Cette prise de conscience initiale n'est donc que l'amorce d'un mouvement plus vaste qui conduit l'individu à redéfinir sa position dans l'univers en passant par le "Connais-toi toi-même" de Socrate. En cherchant le chemin qui mène à soi, "l'homme cherche un territoire, une identité, un accord avec le monde" 37. Plus qu'un refuge, l'intime est une quête.

33 Cours abrégé de Belles Lettres à l'usage du Collège de Montréal, Montréal, De l'imprimerie C. P. Leprohon, 1840, p. 98.

34 Charles Lévesque, "Consolation", La minerve (20 avril 1854), p. 1, reproduit dans Les cuuves de Charles Lévesque écrivain oublié du dix-neuvième siècle (1817-1859), texte établi et annoté par Michel Boucher, op. cit., p. 158 et dans TPCF, tome V, op. cit., $\mathrm{n}^{\circ} 149, \mathrm{p} .333-334$.

35 Joseph-Guillaume Barthe, "Sur la mort prématurée du jeune Petro Adrien Delvecchio, décédé à six ans et deux mois", L'ami du peuple, de l'ordre et des lois (30 mai 1840), p. 1 , reproduit dans TPCF, tome 4, op. cit., $\mathrm{n}^{\circ} 149$, p. 299.

36 Georges Poulet, La conscience critique, Paris, José Corti, 1971, p. 307.

37 Daniel Madelénat, L'intimisme, Paris, Presses universitaires de France, 1989, p. 73. Ces trois éléments reprennent le sempiternel questionnement existentiel : Qui suis-je? (identité), Où vis-je? (territoire), Où vais-je? (accord avec le monde). 